\title{
The gut anaerobe Faecalibacterium prausnitzii uses an extracellular electron shuttle to grow at oxic-anoxic interphases
}

\author{
M Tanweer Khan ${ }^{1}$, Sylvia H Duncan ${ }^{2}$, Alfons JM Stams ${ }^{3}$, Jan Maarten van Dijl ${ }^{1}$, \\ Harry J Flint ${ }^{2}$ and Hermie JM Harmsen ${ }^{1}$ \\ ${ }^{1}$ Department of Medical Microbiology, University Medical Center Groningen, University of Groningen, \\ Groningen, The Netherlands; ${ }^{2}$ Microbial Ecology Group, Rowett Institute of Nutrition and Health, \\ University of Aberdeen, Aberdeen, UK and ${ }^{3}$ Laboratory of Microbiology, Wageningen University, \\ Wageningen, The Netherlands
}

\begin{abstract}
Faecalibacterium prausnitzii is one of the most abundant bacteria in the human gut ecosystem and it is an important supplier of butyrate to the colonic epithelium. Low numbers of faecalibacteria have been associated with inflammatory bowel disease. Despite being extremely oxygen sensitive, $F$. prausnitzii is found adherent to the gut mucosa where oxygen diffuses from epithelial cells. This paradox is now explained on the basis of gas tube experiments, flavin-dependent reduction of 5,5'-dithiobis-2-nitrobenzoate and microbial fuel cell experiments. The results show that F. prausnitzii employs an extracellular electron shuttle of flavins and thiols to transfer electrons to oxygen. Both compounds are present in the healthy human gut. Our observations may have important implications for the treatment of patients with Crohn's disease, for example, with flavin- or antioxidant rich diets, and they provide a novel key insight in host-microbe interactions at the gut barrier.
\end{abstract}

The ISME Journal (2012) 6, 1578-1585; doi:10.1038/ismej.2012.5; published online 23 February 2012 Subject Category: microbial ecology and functional diversity of natural habitats

Keywords: Faecalibacterium prausnitzii; extracellular electron transfer; exogenous electron mediator; riboflavin; thiols; gut-health

\section{Introduction}

Microbial communities in the large intestine have vital roles in the regulation of gut health and pathogenesis of disease. Dietary fibers such as resistant starch, plant cell wall materials and oligosaccharides that escape digestion by host enzymes reach the colon and serve as substrates for microbial fermentation (Saulnier et al., 2009). These plant fibers have a major impact on the gut microbial ecosystem as they serve as growth substrates for gut bacteria and are fermented to short chain fatty acids (SCFAs) (Mahowald et al., 2009). Moreover, they are rich sources of polyphenols that can act as redox mediators (Manach et al., 2004). Due to intense microbial metabolic activity the oxygen tension in the gut lumen is considerably lower when compared to that of the gut mucosa (Due et al., 2003), which possesses a sufficiently high oxygen tension to

Correspondence: HJM Harmsen, Department of Medical Microbiology, University Medical Center Groningen, PO Box 30001, 9700 RB Groningen, The Netherlands.

E-mail: h.j.m.harmsen@med.umcg.nl

Received 26 September 2011; revised 10 January 2012; accepted 11 January 2012; published online 23 February 2012 inhibit growth of the strict anaerobes that are favored in the lumen (Hill, 1995). The major non-gaseous end products of fermentation formed in the colon are SCFAs, including acetate, propionate and butyrate (Topping and Clifton, 2001), that are important modulators of gut health. In particular, butyrate is the preferred energy source for the colonic mucosa and has a role in the protection against colitis and colorectal cancers (Hamer et al., 2009).

Recently, Faecalibacterium prausnitzii, an acetate-consuming and butyrate-producing gut microbe has attracted great interest because of its potentially important role in promoting gut health (Sokol et al., 2008; Louis and Flint, 2009). The most likely pathway utilized by this organism to produce various fatty acids including butyrate from the main carbon source glucose is shown in Figure 1. $F$. prausnitzii is one of the most abundant human colonic bacteria with numbers ranging from $5-20 \%$ of the total microbiota in stools of healthy individuals (Tap et al., 2009; Walker et al., 2011). Moreover, low counts of $F$. prausnitzii have been correlated with several pathological disorders, such as Crohn's disease (Sokol et al., 2009; Willing et al., 2009). Habitually, faecalibacteria are found within the fecal mass but they are also present in the 


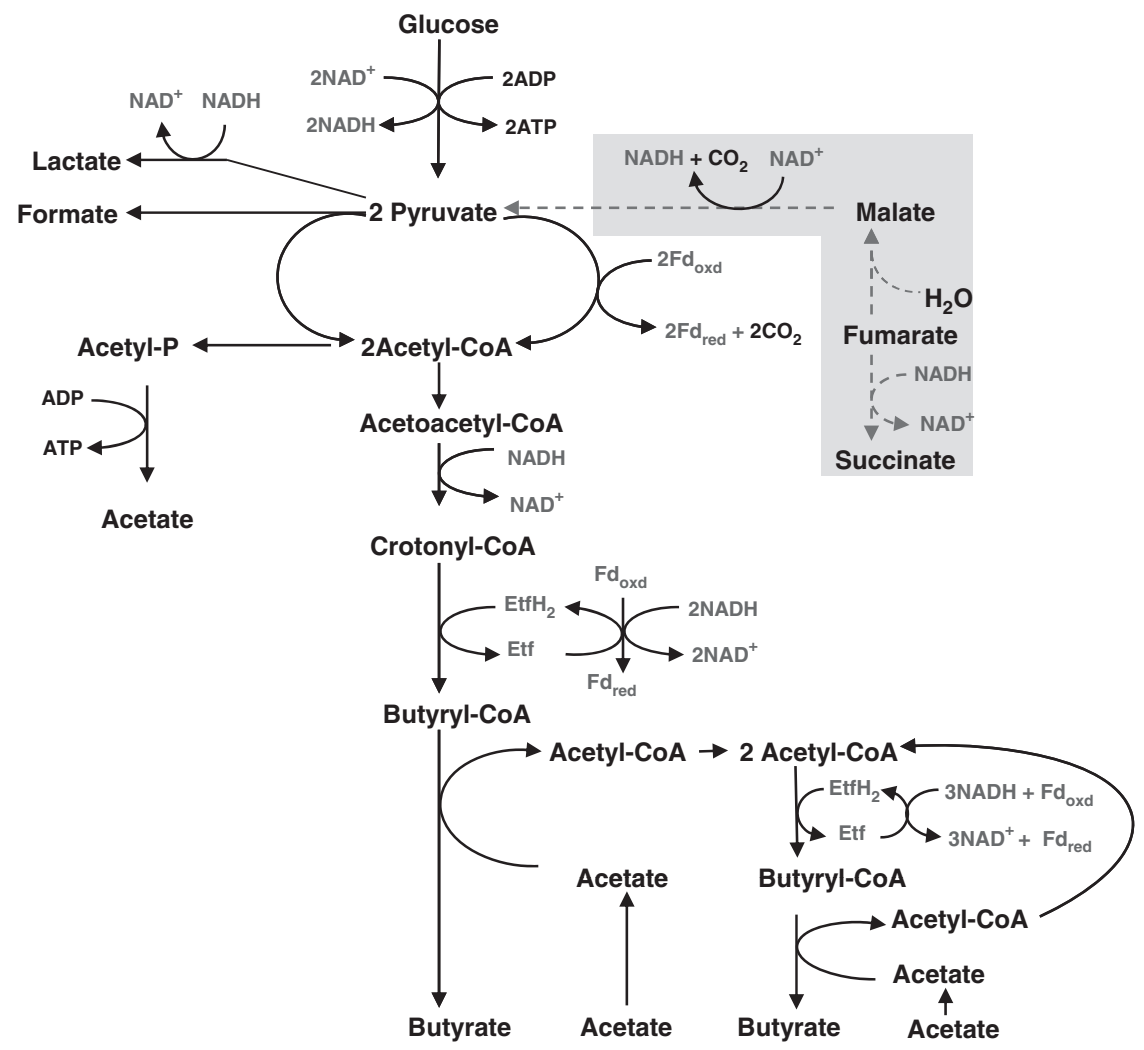

Figure 1 Presumed pathways for the production of butyrate and other short-chain fatty acids from the glucose utilized by $F$. prausnitzii strains. The diagram also depicts the net acetate consumption and cycling of general intracellular electron carriers (blue). Adapted from diagrams described by Herrmann et al. (2008) and by Seedorf et al. (2008). An alternative pathway for the use of fumarate as terminal electron acceptor and carbon source is indicated in the grey box with red dotted arrows. The colour reproduction of this figure is available at the ISME Journal online.

adjacent mucosa where there is oxygen influx from the gut epithelium (Swidsinski et al., 2008; Van den Abbeele et al., 2011). To date, F. prausnitzii has been considered a strict anaerobe that loses its viability within 2 min after exposure to ambient air (Duncan et al., 2002). It was thus unclear how such a strict anaerobe could exist in regions of the colon with relatively high oxygen tensions. Our present studies were aimed at unraveling the factors that enable the survival of $F$. prausnitzii in oxygenated niches within the human colon.

\section{Materials and methods}

Bacterial strains and culturing conditions

F. prausnitii strain A2-165 (DSM 17677) was described previously (Duncan et al., 2002) and F. prausnitzii strain HTF-F was isolated from human feces and identified by $16 \mathrm{~S}$ rRNA sequencing. Roseburia inulinivorans A2-194 (DSM16841) (Duncan et al., 2006), Bacteroides fragilis ATCC 25285, Escherichia coli ATCC 25922 and Bifidobacteriium animalis ATCC 25527 were used as control strains. All strains were maintained at $37^{\circ} \mathrm{C}$ on yeast extract, casitone, fatty acid and glucose medium (YCFAG) in an anaerobic tent (Duncan et al., 2002; Lopez-Siles et al., 2012). For microbial fuel cell experiments, the bacterial cells were grown in $50 \mathrm{ml}$ of YCFAG broth to an optical density at $600 \mathrm{~nm}$ $\left(A_{600}\right)$ of $\sim 0.8$. Cells were harvested by centrifugation, washed in $50 \mathrm{~mm}$ potassium phosphate buffer and resuspended in $0.5 \mathrm{ml}$ of the same buffer. The resuspended cells were used to inoculate the anode chamber of a microbial fuel cell. All buffers and media were deoxygenated by flushing with oxygenfree nitrogen for $30 \mathrm{~min}$.

Gas tube experiments

To test for growth at different oxygen concentrations, $50 \mu \mathrm{l}$ of a bacterial overnight culture was mixed with $7 \mathrm{ml}$ of molten YCFAG agar medium $\left(\sim 38^{\circ} \mathrm{C}\right)$ and poured into $250 \mathrm{~mm} \times 18 \mathrm{~mm}$ glass tubes that were sealed at both ends with gas-tight butyl rubber plugs. Sterile air was blown in at one end, while the other end contained $\mathrm{N}_{2}(81 \%), \mathrm{H}_{2}$ $(11 \%)$ and $\mathrm{CO}_{2}(8 \%)$. The presence or absence of a growth rim was observed after $16-20 \mathrm{~h}$ at $37^{\circ} \mathrm{C}$. The basal medium used to test conditions for growth rim formation was YCFAG medium without hemin, resazurin, cysteine, yeast extract, acetate and other SCFAs. To determine the effect of glutathione on growth rim formation, $0.14 \%$ glutathione was added to riboflavin-containing basal medium instead of cysteine. All tested chemicals in the different 
experiments were added separately at the same concentration as in YCFAG medium. Riboflavin was added in the concentration of $0.75 \mu \mathrm{g} \mathrm{ml}^{-1}$. SCFA production profiles of $F$. prausnitzii strains were assessed by growing the cultures in YCFAG10 medium in which the glucose concentration was reduced to $10 \mathrm{mM}$.

\section{Oxygen consumption assays}

Oxygen consumption assays were conducted in $35 \mathrm{ml}$ crimp cap vials provided with butyl stoppers containing $5 \mathrm{ml}$ of YCFAG medium. Medium was prepared and added in a strictly anaerobic oxygenfree nitrogen environment and $15 \mathrm{ml}$ of sterile air was injected after inoculation. The residual oxygen in the gas phase was measured by gas chromatography after $48 \mathrm{~h}$ incubation, while the spent broth was used for SCFA analysis. In these vials oxygen acts as terminal electron acceptor. Vials having nitrogen in the gas phase served as control for fatty acid production under anaerobic conditions. Additionally, sodium fumarate $(55 \mathrm{mM})$ was used as terminal electron acceptor in YCFAG10 medium and, after $24 \mathrm{~h}$ of incubation, the residual broth was used to determine the fatty acid production profile.

\section{Microbial fuel cell experiments}

A two-chambered microbial fuel cell with working volumes of $65 \mathrm{ml}$ in the cathode and anode chambers was constructed as described by Park and Zeikus (2000). A CMI-7000S cation exchange membrane (Membranes International INC., Ringwood, NJ, USA) was used as a septum between the two chambers. A $0.5-\mathrm{mm}$ platinum wire was used as cathode with $100 \mathrm{mM}$ potassium-phosphate buffer as catholyte. A plain graphite plate $(5 \mathrm{~cm} \times 1.5 \mathrm{~cm} \times 0.2 \mathrm{~cm})$ was used as anode. Furthermore, the anode chamber contained $50 \mathrm{~mm}$ potassium-phosphate buffer as anolyte, $0.1 \mathrm{M}$ glucose and an $\mathrm{Ag} / \mathrm{AgCl}$ reference electrode. The whole system was maintained at $37^{\circ} \mathrm{C}$, and the anode chamber was continuously flushed with nitrogen gas. A voltage of $-100 \mathrm{mV}$ versus the $\mathrm{Ag} / \mathrm{AgCl}$ reference electrode was applied on the anode, and the generated current was monitored at $30 \mathrm{~s}$ intervals using a potentiostat (model \# 600C, CH Instruments, Austin, TX, USA). After energizing the bacterial cells with glucose for $10 \mathrm{~min}, 200 \mu \mathrm{M}$ riboflavin was injected into the anode chamber.

\section{Flavin-mediated DTNB reduction assay and sugar estimation}

To test for 5, 5'-dithiobis-(2-nitrobenzoate) (DTNB, Sigma Aldrich, St Louis, MO, USA) reduction, $1 \mathrm{ml}$ aliquots of overnight cultures of $F$. prausnitzii A2-165 or HTF-F in YCFAG medium were harvested by centrifugation and washed twice with anaerobic potassium phosphate buffer solution (50 mM).
The cells were then added to a reaction mixture containing $50 \mathrm{mM}$ potassium phosphate buffer, $60 \mu \mathrm{M}$ $\mathrm{CaCl}_{2}, 36 \mu \mathrm{M} \mathrm{MgCl}_{2}, 1.3 \mathrm{mM}$ DTNB, $15 \mathrm{mM} \mathrm{NaCl}$ and $3.3 \mathrm{mM}$ sodium acetate. The reaction mixture was incubated anaerobically for $30 \mathrm{~min}$ at $37^{\circ} \mathrm{C}$ with or without riboflavin $\left(0.75 \mu \mathrm{g} \mathrm{ml}^{-1}\right)$, flavin adenine dinucleotide $\left(0.75 \mu \mathrm{g} \mathrm{ml}^{-1}\right)$ or yeast extract $(0.2 \%)$, and the development of yellow color was monitored as an increased absorbance at $420 \mathrm{~nm}\left(\mathrm{~A}_{420}\right)$ using UV-Visible spectroscopy. The results were expressed as fold increase in $\mathrm{A}_{420}$ relative to the absorbance without added flavins or yeast extract. The glucose consumption was measured using the 3,5-dinitrosalicylic acid (DNSA) method (Miller, 1959).

\section{Chromatography}

A HPLC Ion Chromatography system (Metrohm AG, Herisau, Switzerland) with a conductivity detector was used for measuring fatty acid concentrations including lactate, formate, butyrate, propionate and acetate. A 6.1005.200 Metrosep Organic Acids column (Metrohm AG) with $5 \mu \mathrm{m}$ particle size and dimensions of $7.8 \mathrm{~mm} \times 250 \mathrm{~mm}$ was used for fatty acid separation. The mobile phase was composed of $5 \mathrm{mM} \mathrm{HNO}_{3}$ and acetonitrile with a v/v ratio of 98:2, respectively, and a flow rate of $0.6 \mathrm{ml} \mathrm{min}^{-1}$ was applied. Fatty acid estimation in samples having sodium fumarate as terminal electron acceptor was conducted on a HPLC system (Waters Chromatography B.V., Etten-Leur, The Netherlands) with a refractive index detector. Separation was achieved using a Varian Metacarb 67H, $300 \mathrm{~mm} \times 7.8 \mathrm{~mm}$ column (MetaChem Technologies Inc., Torrance, CA, USA) at room temperature in isocratic mode at a flow rate of $0.6 \mathrm{ml} \mathrm{min}^{-1}$. A guard column of the same phase was placed in front of the analytical column. The eluent was $0.5 \mathrm{mM}$ sulfuric acid (AR grade, Sigma Aldrich). Oxygen consumption was analyzed by gas chromatography using a Molecular Sieve column 5A 80/100 mesh (Agilent Technologies, Amstelveen, The Netherlands).

\section{Results}

Growth of F. prausnitzii in the presence of oxygen Previous tests indicated extreme oxygen sensitivity of F. prausnitzii cells on agar surfaces to air containing about $20 \%$ oxygen (Duncan et al., 2002). In the present studies, the influence of different gas phases on growth of $F$. prausnitzii was tested under conditions that create an oxygen gradient (Figure 2a). Surprisingly, F. prausnitzii was found to form a growth rim at the oxygenated end (Figure 2b). In contrast, another obligate anaerobic Firmicute that also produces butyrate in the human colon, $R$. inulinivorans A2-194, only grew diffusely throughout the anoxic zone (Figure 2c). The rim formation by $F$. prausnitzii was due to enhanced growth at the junction of the oxidized (pink) and reduced (colorless) zones revealed by resazurin, which was used as 


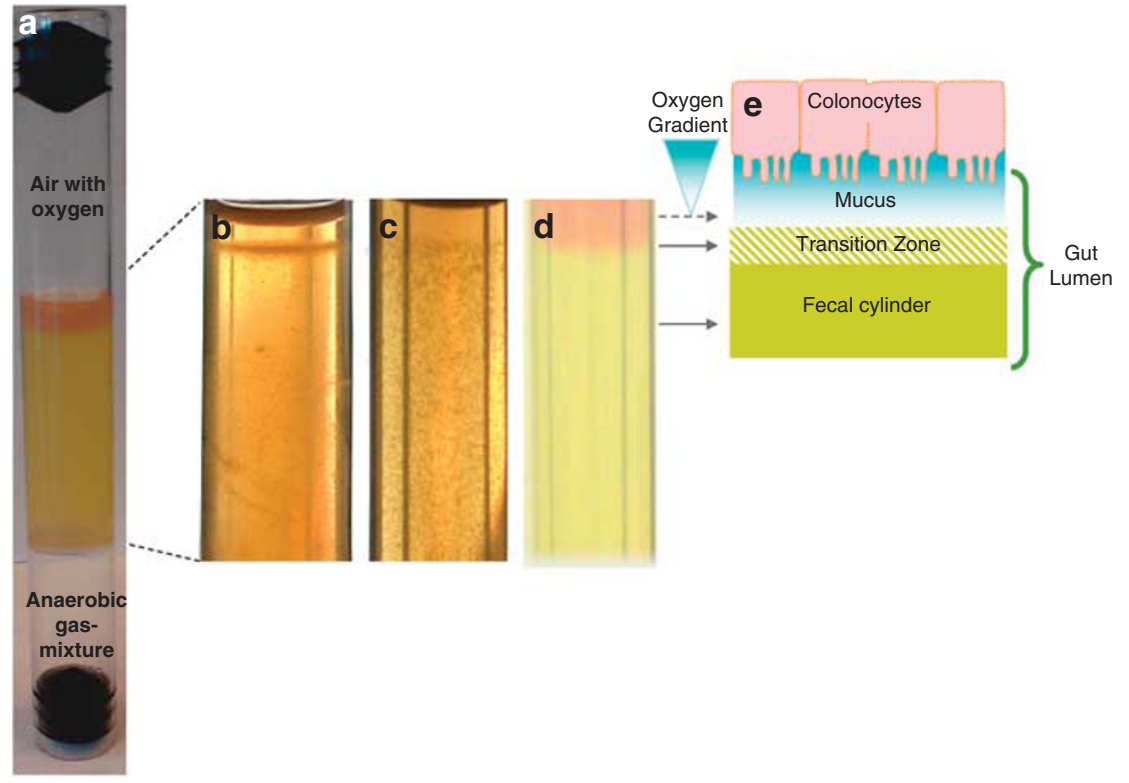

Figure 2 Growth of anaerobic gut bacteria after $16 \mathrm{~h}$ in agar medium with and without an oxygenated gas phase. (a) Gas tube experiments are based on glass tubes with two gas phases at the opposite ends of an agar medium plug. The gas phase at the top is composed of $\sim 5 \mathrm{ml}$ oxygen-containing air and the gas phase at the bottom is an anaerobic gas- mixture of $\sim 81 \% \mathrm{~N}_{2}, \sim 11 \% \mathrm{H}_{2}$ and $\sim 8 \%$ $\mathrm{CO}_{2}$. (b) F. prausnitzii A2-165 showing enhanced growth in a rim at the oxygenated end and moderate growth in small colonies throughout the rest of the agar plug. (c) R. inulinivorans A2-194 showing growth in colonies throughout the agar plug except at the oxygenated zone. (d) Un-inoculated medium showing a color change of resazurin due to oxygen diffusion. (e) Schematic interpretation of the gas tube oxygen gradient on the human gut lumen and mucosal layer (Van den Abbeele et al., 2011). F. prausnitzii can be found in the transition zone or adherent to gut-mucosa (Swidsinski et al., 2008).

a redox indicator (Figure $2 \mathrm{~d}$ ). The oxygenated zone was deeper $(1.5 \mathrm{~cm} \pm 0.2 \mathrm{~cm})$ in uninoculated gas tubes as compared with the inoculated tubes $(0.5 \mathrm{~cm} \pm 0.2 \mathrm{~cm})$. This suggested that oxygen was being consumed by $F$. prausnitzii cells growing in the rim. Eight different isolates of $F$. prausnitzii were tested and all showed the ability to form the growth rim in the oxygenated gas tube zone.

Growth rim formation by F. prausnitzii requires an extracellular flavin-thiol shuttle and a terminal electron acceptor

To understand the mechanism of growth rim formation, basal medium was supplemented with different components. Of the normal components of YCFAG medium (Lopez-Siles et al., 2012), cysteine and yeast extract were both required for growth rim formation by $F$. prausnitzii, but yeast extract could be replaced by riboflavin. However, cysteine alone or in combination with either hemin, resazurin or acetate did not result in the formation of a growth rim. Riboflavin, present in yeast extract was recently reported to be exploited by the fermentative bacterium Lactococcus lactis, as an exogenous redox mediator for extracellular electron transfer (Masuda et al., 2010). To confirm the presumed role of flavins and oxidized disulfides in an extracellular electron shuttle, a 5, 5 '-dithiobis-(2- nitrobenzoate) (DTNB) assay was performed (Figure 3a). Indeed, F. prausnitzii A2165 cells from the mid log phase $\left(\mathrm{OD}_{660} 0.48 \pm 0.1\right)$ were able to use yeast extract or riboflavin as a redox mediator for extracellular electron transfer to DTNB. Reduction of flavins was accompanied by electron transfer to the disulfide bond in DTNB resulting in the yellow-colored compound 2-nitro-5-thiobenzoate $\left(\mathrm{NTB}^{-}\right)$. As shown for strain A2-165, addition of riboflavins, flavin adenine dinucleotide or yeast extract significantly increased the relative folds of absorbance at $420 \mathrm{~nm}$ by $9 \pm 0.2,7 \pm 0.2$ and $6.5 \pm 0.4$, respectively, as compared to samples without added flavins. This implies that flavins are a prerequisite for shuttling electrons. The flavin-thiol shuttling is a feature of living cells, as cells killed with chloroform did not reduce DTNB. We therefore propose that flavins and thiols act as redox mediators in the human gut and that electrons are finally transferred to oxygen (Figure 3b). Accordingly, F. prausnitzii rim formation in basal medium with riboflavin was also facilitated by glutathione, which is a primary thiolcontaining antioxidant in the gut (Loguercio, 2003; Burrin and Stoll, 2007). To measure the consumption of oxygen, cells were grown in a vial containing yeast extract, casitone, SCFAs including acetate and glucose (YCFAG) broth with oxygen in the gas phase. Indeed, under these conditions a considerable amount of oxygen was consumed per mmol of glucose utilized (Figures 4a and b). Interestingly, our growth experiments showed that faecalibacteria are also capable of efficiently using fumarate as an alternative electron acceptor if no oxygen is present (Figures 1, 4a and b). 

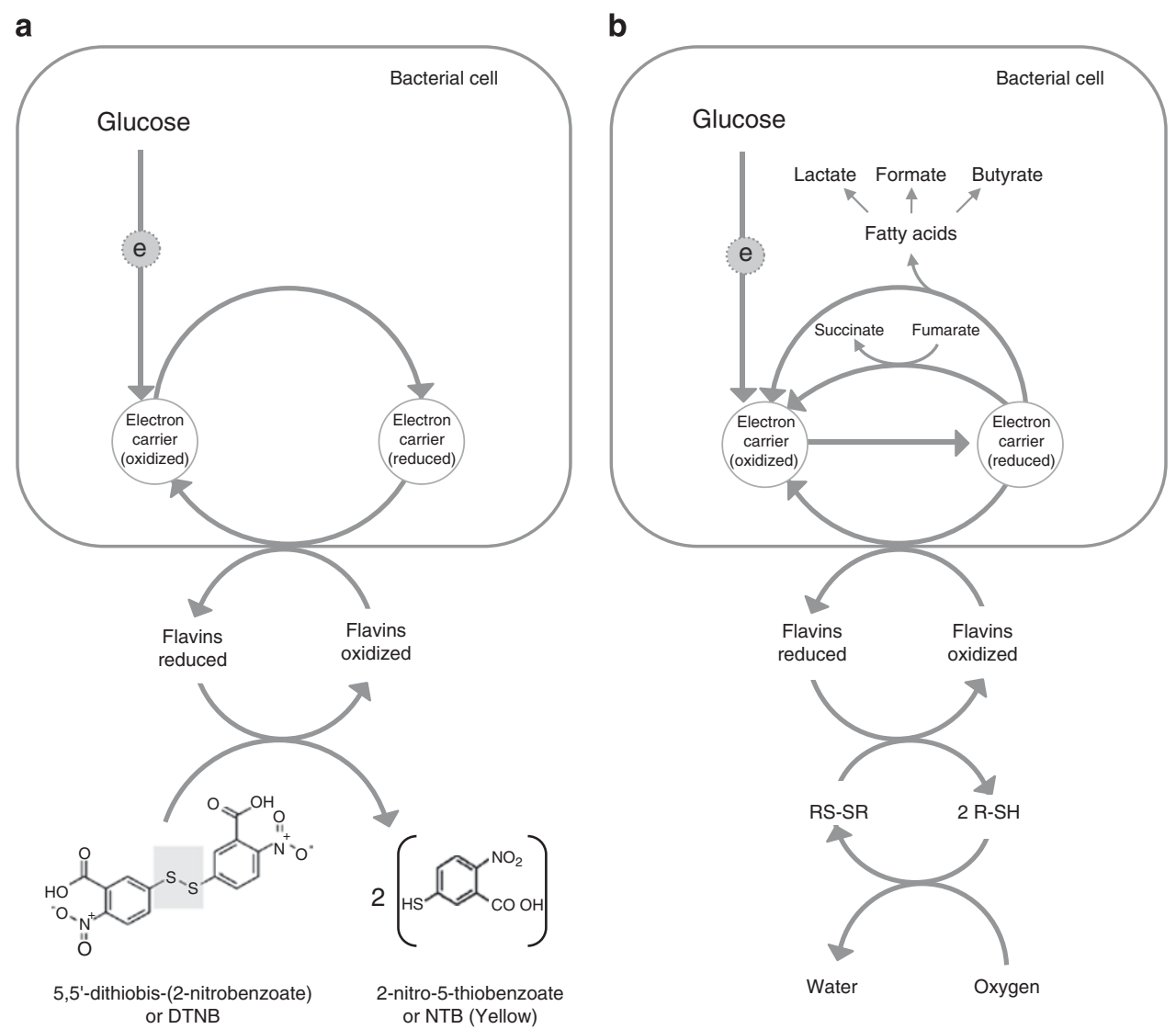

Figure 3 The faecalibacterial extracellular flavin-thiol electron shuttle. (a) Bacterial cell, flavin-thiol electron shuttle experiment. In this experiment the bacterial cells (schematically represented) reduce riboflavin with the help of intracellular electron carriers (see Figure 1). The electrons are subsequently transferred from riboflavin to the disulfide bond in DTNB, resulting in the yellow-colored compound $\mathrm{NTB}^{-}$. No yellow color is formed when the cells are incubated with DTNB in the absence of riboflavins. (b) Model for extracellular electron transfer to oxygen via a flavin-thiol redox shuttle. In the absence of terminal electron acceptor, reducing equivalents are used to produce SCFAs such as lactate, formate or butyrate. If fumarate is available during anaerobic growth, a membraneassociated fumarate reductase transfers electrons to fumarate, resulting in the production of succinate.

\section{Riboflavin is essential in extracellular electron shuttling}

To validate the proposed role of riboflavin in extracellular electron shuttling, microbial fuel cell experiments were performed with resting cells of $F$. prausnitzii. As shown in Figure 5 for the F. prausnitzii strain A2-165, no current was generated when the cells were energized with glucose. However, when riboflavin was added to the pre-energized cells in the anode chamber, a rapidly increasing current was observed that reached a plateau level of about $6 \mathrm{~mA}$ within 5-6 min after the addition of riboflavin (Figure 5). Importantly, no current was observed when riboflavin was added to anode chambers without cells of $F$. prausnitzii, anode chambers with glucose-starved or chloroform-treated cells of $F$. prausnitzii, or anode chambers that were inoculated with cells of $B$. animalis, B. fragilis, E. coli or R. inulinivorans. These findings show unambiguously that riboflavin is required for the extracellular shuttling of electrons by $F$. prausnitzii cells that metabolize glucose aerobically.
The influence of extracellular electron shuttling on SCFA production

Butyrate is generally regarded as the major fermentative end product of $F$. prausnitzii under anaerobic conditions (Figure 1) (Louis and Flint, 2009; Benus et al., 2010). In view of the importance of butyrate for gut health, we investigated whether butyrate production might be altered under the newly identified growth conditions for $F$. prausnitzii. To this end, the molar yields of butyrate per mole of glucose for two metabolically different strains of F. prausnitzii were compared (Lopez-Siles et al., 2012) under anaerobic conditions in the presence or absence of fumarate, and under aerobic conditions (carbon balances and molar yields are presented in Supplementary Table 1). Although strains A2-165 and HTF-F are closely related phylogenetically, they differ in growth rates, as the strain A2-165 grows faster than strain HTF-F. These strains also differ in detail in product formation as strain A2-165 produces four-times more formate when grown on anaerobic YCFAG medium when compared with strain HTF-F. For strain A2-165, a decreased 


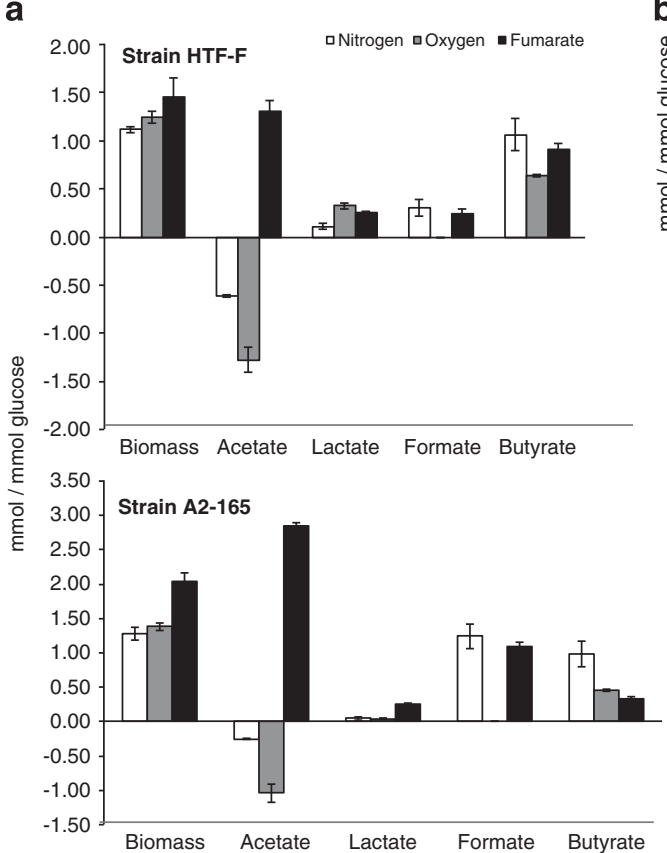

b

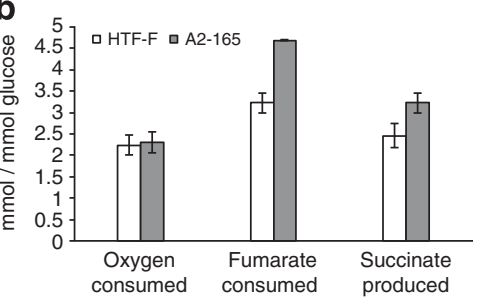

Figure 4 (a) Biomass and organic acids produced or acetate consumed (mmoles) per mmole of glucose by two different strains of F. prausnitzii. The strains were cultivated in YCFAG medium with a nitrogen or oxygen gas phase. Alternatively, fumarate was added to the medium of the strains cultivated with a nitrogen head. Note that both oxygen and fumarate can serve as terminal electron acceptors. (b) Oxygen or fumarate consumption per mmol of glucose, and succinate production by two isolates of $F$. prausnitzii. The data presented here is the mean of three independent replicates.

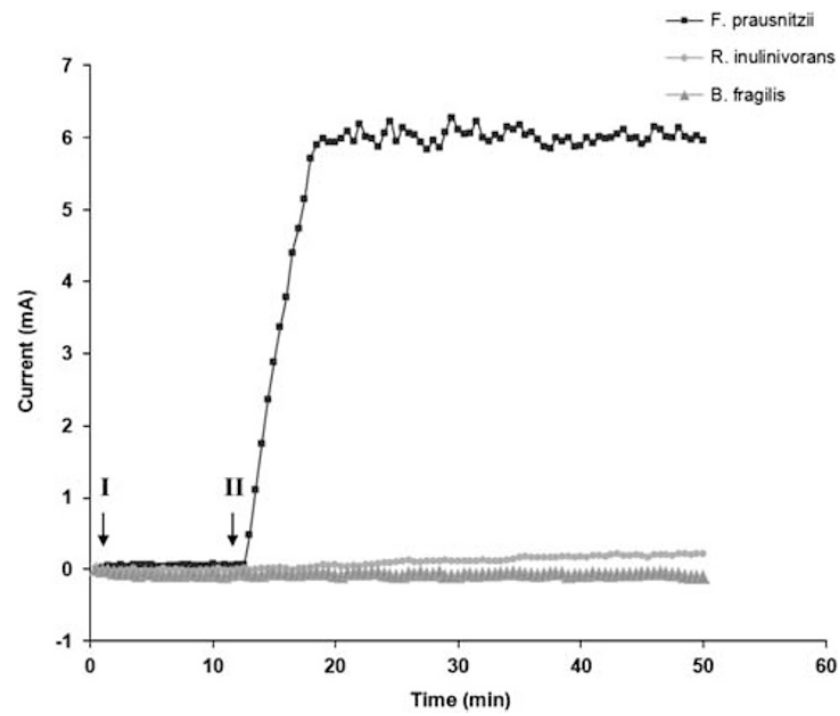

Figure 5 F. prausnitzii requires riboflavin for electron transfer to the anode of a microbial fuel cell. The diagram shows current production profiles for the human gut bacteria $F$. prausnitzii, $B$. fragilis and $R$. inulinivorans. (I) Bacterial cells were added to the anode chamber containing potassium phosphate buffer $(50 \mathrm{mM})$ and glucose $(0.1 \mathrm{M})$. A voltage of $-100 \mathrm{mV}$ versus $\mathrm{Ag} / \mathrm{AgCl}$ reference electrode was applied and the current was measured at $30 \mathrm{~s}$ intervals. (II) After $10 \mathrm{~min}$ of incubation at $37^{\circ} \mathrm{C}$, $200 \mu \mathrm{M}$ of riboflavin was injected as an extracellular redox mediator into the anode chamber. Under these conditions only $F$. prausnitzii generates a measurable current wave.

butyrate production was observed under oxygenated growth conditions or anoxic conditions with fumarate present (Figure 4a). This must relate to an electron transfer shift from fermentation, that is, SCFA production, to 'aerobic respiration' via the flavin-cysteine redox shuttle or to the anaerobic reduction of fumarate by transferring electrons from $\mathrm{NADH}$ to succinate while regenerating $\mathrm{NAD}^{+}$ (Figure 1). In contrast, strain HTF-F did not show a significant decrease in butyrate production when fumarate was used as terminal electron acceptor. This behavior might be due to physiological differences between the tested strains (Lopez-Siles et al., 2012). In the presence of fumarate both strains produced malate and acetate, indicating that fumarate can also serve as carbon source. The elevated acetate formation in strain A2-165 may be explained by decreased acetate cycling (Louis and Flint, 2009). Thus, faecalibacteria metabolize glucose to acetate, $\mathrm{CO}_{2}$ and electrons, which are disposed of via SCFA formation. Alternatively, the electrons are either transferred to oxygen via extracellular redox mediators or to fumarate, which results in succinate production. Incubations with ${ }^{13} \mathrm{C}$ acetate in the presence of oxygen did not lead to the enrichment of ${ }^{13} \mathrm{C}$ in $\mathrm{CO}_{2}$ (data not shown), which implies that there was no operational TCA cycle under the conditions tested.

\section{Discussion}

In the present studies, we have shown that cells of $F$. prausnitzii can grow in the presence of oxygen, provided that their growth medium contains flavins and cysteine or glutathione. Based on our findings, 
we propose that in the presence of oxygen the free thiols of cysteine or glutathione are oxidized to disulfide bonds that subsequently act as electron acceptors, whereas the flavins act as redox mediators for faecalibacterial cells as shown in Figures 3a and $b$. The rapid shuttling of flavins through the cell envelope is a rather specialized phenomenon, as Pseudomonas species and Escherichia coli produce riboflavin but do not utilize this compound for extracellular electron transfer (von Canstein et al., 2008). Importantly, our findings give a rationale for the growth and survival of $F$. prausnitzii at the oxygenated fecal-mucosal interface of the human gut (Swidsinski et al., 2008).

Consumed dairy products, plant foliage, fruits and fibers are the main sources of flavin in the gut, whereas thiols can also be obtained by various dietary sources such as egg yolk, dairy products and grains (Dangour, 2010). Additionally, the gut mucosa harbors considerable amounts of thiols that are secreted by colonocytes and act as antioxidants (Keshavarzian, 1992; Loguercio, 2003). Several studies have demonstrated an oxidative surge in colitis that is associated with decreased concentrations of thiols (Aw, 2003; Seril et al., 2003). Oral supplementation of these compounds together with flavins is therefore likely to be useful in controlling the progression of colitis (Loguercio, 2003; Dryden et al., 2005; Kim et al., 2009). This also indicates that such compounds in the gut lumen promote 'gut health' (Kau et al., 2011) and that they may be utilized by anaerobic bacteria in niches, where an oxygen gradient exists such as the gut mucosa.

Extracellular electron transfer by $F$. prausnitzii provides a very plausible mechanistic explanation for the survival of certain anaerobes in oxygenated environments where appropriate redox mediators are available. It remains to be established whether other human colonic anaerobes possess such a capability for extracellular electron transfer, although this was not evident for the butyrate producer $R$. inulinivorans (Figure 2c), and other gut bacteria like $B$. animalis, $B$. fragilis or E. coli. Furthermore, our results may provide new insights into clinical conditions such as Crohn's disease. This disease is characterized by aberrant mucosa, thiol depletion and oxidative stress leading to an altered microbiota, for instance reduction in F. prausnitzii numbers, which will most likely impact on the pathogenesis (Keshavarzian, 1992; Sokol et al., 2008; Kim et al., 2009). Taken together, the present findings highlight the potential for the flavin and antioxidant status of the diet to have a major influence on beneficial bacteria and their distribution in the human gut and hence on gut health.

\section{Acknowledgements}

We would like to thank Ton van Gelder and Gerrie Stob for fatty acid analysis, Gerald Lobley and Eric Milne for analysis of the fate of ${ }^{13} \mathrm{C}$ acetate and Wesley Browne for the help with the microbial fuel cell. MTK was supported by a grant from the Graduate School GUIDE of the University of Groningen. SHD and HJF were supported by the Scottish Government Rural and Environment Research and Analysis Directorate. AJMS was supported by the Chemical Sciences division of the Netherlands Organization for Scientific Research (NWO).

\section{References}

Aw TY. (2003). Cellular redox: a modulator of intestinal epithelial cell proliferation. News Physiol Sci 18: 201-204.

Benus RF, van der Werf TS, Welling GW, Judd PA, Taylor MA, Harmsen HJ et al. (2010). Association between Faecalibacterium prausnitzii and dietary fibre in colonic fermentation in healthy human subjects. $\mathrm{Br}$ J Nutr 29: 1-8.

Burrin DG, Stoll B. (2007). Emerging aspects of gut sulfur amino acid metabolism. Curr Opin Clin Nutr Metab Care 10: 63-68.

Dangour AD. (2010). Nutrition-related health effects of organic foods: a systematic review. Am J Clin Nutr 92: 203-210.

Dryden Jr GW, Deaciuc I, Arteel G, McClain CJ. (2005). Clinical implications of oxidative stress and antioxidant therapy. Curr Gastroenterol Rep 7: 308-316.

Due VL, Bonde J, Kann T, Perner A. (2003). Extremely low oxygen tension in the rectal lumen of human subjects. Acta Anaesthesiol Scand 47: 372.

Duncan SH, Aminov RI, Scott KP, Louis P, Stanton TB, Flint HJ. (2006). Proposal of Roseburia faecis sp. nov., Roseburia hominis sp. nov. and Roseburia inulinivorans sp. nov., based on isolates from human faeces. Int $J$ Syst Evol Microbiol 56: 2437-2441.

Duncan SH, Hold GL, Harmsen HJ, Stewart CS, Flint HJ. (2002). Growth requirements and fermentation products of Fusobacterium prausnitzii, and a proposal to reclassify it as Faecalibacterium prausnitzii gen. nov., comb. nov. Int J Sys Evol Microbiol 52: 2141-2146.

Hamer HM, Jonkers DM, Bast A, Vanhoutvin SA, Fischer MA, Kodde A et al. (2009). Butyrate modulates oxidative stress in the colonic mucosa of healthy humans. Clin Nutr 28: 88-93.

Herrmann G, Jayamani E, Mai G, Buckel W. (2008). Energy conservation via electron-transferring flavoprotein in anaerobic bacteria. J Bacteriol 190: 784-791.

Hill MJ. (1995). The Normal Gut Bacterial Flora. Taylor \& Francis: London, 1-18.

Kau AL, Ahern PP, Griffin NW, Goodman AL, Gordon JI. (2011). Human nutrition, the gut microbiome and the immune system. Nature 474: 327-336.

Keshavarzian A. (1992). Excessive production of reactive oxygen metabolites by inflamed colon: analysis by chemiluminescence probe. Gastroenterology 103: 177-185.

Kim CJ, Kovacs-Nolan J, Yang C, Archbold T, Fan MZ, Mine Y. (2009). L-cysteine supplementation attenuates local inflammation and restores gut homeostasis in a porcine model of colitis. Biochim Biophys Acta 1790: 1161-1169. 
Loguercio C. (2003). Glutathione supplementation improves oxidative damage in experimental colitis. Dig Liver Dis 35: 635-641.

Lopez-Siles M, Khan TM, Duncan SH, Harmsen HJ, Garcia-Gil LJ, Flint HJ. (2012). Cultured representatives of two major phylogroups of human colonic Faecalibacterium prausnitzii can utilize pectin, uronic acids, and host-derived substrates for growth. Appl Environ Microbiol 78: 420-428.

Louis P, Flint HJ. (2009). Diversity, metabolism and microbial ecology of butyrate-producing bacteria from the human large intestine. FEMS Microbiol Lett 94: 1-8.

Mahowald MA, Rey FE, Seedorf H, Turnbaugh PJ, Fulton RS, Wollam A et al. (2009). Characterizing a model human gut microbiota composed of members of its two dominant bacterial phyla. Proc Natl Acad Sci USA 106: 5859-5864.

Manach C, Scalbert A, Morand C, Rémésy C, Jiménez L. (2004). Polyphenols: food sources and bioavailability. Am J Clin Nutr 79: 727-747.

Masuda M, Freguia S, Wang YF, Tsujimura S, Kano K. (2010). Flavins contained in yeast extract are exploited for anodic electron transfer by Lactococcus lactis. Bioelectrochemistry 78: 173-175.

Miller GL. (1959). Use of dinitrosalicylic acid reagent for determination of reducing sugar. Anal Chem 31: $426-428$.

Park DH, Zeikus JG. (2000). Electricity generation in microbial fuel cells using neutral red as an electronophore. Appl Environ Microbiol 66: 1292-1297.

Saulnier DM, Kolida S, Gibson GR. (2009). Microbiology of the human intestinal tract and approaches for its dietary modulation. Curr Pharm Des 15: 1403-1414.

Seedorf H, Fricke WF, Veith B, Brüggemann H, Liesegang H, Strittmatter A et al. (2008). The genome of Clostridium kluyveri, a strict anaerobe with unique metabolic features. Proc Natl Acad Sci USA 105: 2128-2133.

Seril DN, Liao J, Yang GY, Yang CS. (2003). Oxidative stress and ulcerative colitis-associated carcinogenesis: studies in humans and animal models. Carcinogenesis 24: 353-362.

Sokol H, Pigneur B, Watterlot L, Lakhdari O, BermúdezHumarán LG, Gratadoux JJ et al. (2008). Faecalibacterium prausnitzii is an anti-inflammatory commensal bacterium identified by gut microbiota analysis of Crohn disease patients. Proc Natl Acad Sci 105: 16731-16736.

Sokol H, Seksik P, Furet JP, Firmesse O, Nion-Larmurier I, Beaugerie L et al. (2009). Low counts of Faecalibacterium prausnitzii in colitis microbiota. Inflamm Bowel Dis 15: 1183-1189.

Swidsinski A, Loening-Baucke V, Verstraelen H, Osowska S, Doerffel Y. (2008). Biostructure of fecal microbiota in healthy subjects and patients with chronic idiopathic diarrhea. Gastroenterology 135: 568-579.

Tap J, Mondot S, Levenez F, Pelletier E, Caron C, Furet JP et al. (2009). Towards the human intestinal microbiota phylogenetic core. Environ Microbiol 11: 2574-2584.

Topping DL, Clifton PM. (2001). Short-chain fatty acids and human colonic function: roles of resistant starch and nonstarch polysaccharides. Physiol Rev 81: 1031-1064.

Van den Abbeele P, Van de Wiele T, Verstraete $W$, Possemiers S. (2011). The host selects mucosal and luminal associations of co-evolved gut microbes: a novel concept. FEMS Microbiol Rev 35: 681-704.

von Canstein H, Ogawa J, Shimizu S, Lloyd JR. (2008). Secretion of flavins by Shewanella species and their role in extracellular electron transfer. Appl Environ Microbiol 74: 615-623.

Walker AW, Ince J, Duncan SH, Webster LM, Holtrop G, $\mathrm{Ze} \mathrm{X}$ et al. (2011). Dominant and diet-responsive groups of bacteria within the human colonic microbiota. ISMEJ 5: 220-230.

Willing B, Halfvarson J, Dicksved J, Rosenquist M, Järnerot G, Engstrand L et al. (2009). Twin studies reveal specific imbalances in the mucosa-associated microbiota of patients with ileal Crohn's disease. Inflamm Bowel Dis 15: $653-660$.

Supplementary Information accompanies the paper on The ISME Journal website (http://www.nature.com/ismej) 